\title{
Hyperhomocysteinemia and Central Retinal Vein Occlusion in Iranian Population
}

\author{
Sasan Moghimi, MD, ${ }^{1}$ Zahra Najmi, ${ }^{2}$ Hooshang Faghihi, $M D^{3}$ \\ Reza Karkhaneh, $\mathrm{MD}^{4}$ Mohammad-Sadegh Farahvash, $M D^{4}$
}

\begin{abstract}
Purpose: To evaluate total plasma homocysteine level during the acute phase of central retinal vein occlusion (CRVO) compared with a matched healthy group in Iranian population, and determine whether hyperhomocysteinemia is also a risk factor for CRVO.

Methods: A study group contains 54 patients presenting with CRVO in recent one month, acute phase of the decease, was compared for fasting total plasma homocysteine level with a matched control group of 51 patients evaluated in the same clinic for a non retinal disease diagnosis.

Results: The mean total plasma homocysteine level was $14.76 \pm 7.67 \mu \mathrm{mol} / \mathrm{l}$ in cases, and $11.42 \pm 3.74 \mu \mathrm{mol} / \mathrm{l}$ in control subjects. It shows a significant difference $(p=0.005)$ in mean plasma homocysteine level between the cases and control group. Odds ratio of CRVO for individuals with hyperhomocysteinemia was $2.88(95 \% \mathrm{Cl}=1.08-7.71$ and $\mathrm{p}=0.03)$. The overall multivariableadjusted odds of CRVO in participants with plasma homocysteine level above $15 \mu \mathrm{mol} / \mathrm{l}$ was 4.71 (95\% Cl=1.46-15.19 and $\mathrm{p}=0.009)$ Hyperhomocysteinemia was not statistically different in each age group (<60 years: $27 \%, 61-70$ years: $33.3 \%, 71-80$ years: $31.6 \%,>81$ years: $33.3 \%$, Chi-square test, $p=0.98$ )

Conclusion: Elevated total plasma homocysteine level is an independent risk factor for CRVO in Iranian population. In addition to an evaluation of all conventional cardiovascular risk factors, measurement of total homocysteine for evidence of hyperhomocysteinemia may be important in the initial investigation and management of patients with CRVO.
\end{abstract}

Keywords: central retinal vein occlusion, homocysteine, hyperhomocysteinemia, high-performance liquid chromatography

Iranian Journal of Ophthalmology 2008;20(1):9-15

1. Assistant Prof. of Ophthalmology, Eye Research Center, Farabi Eye Hospital, Medical Sciences/University of Tehran

2. Medical Student, Farabi Eye Hospital, Medical Sciences/University of Tehran

3. Associate Prof. of Ophthalmology, Eye Research Center, Farabi Eye Hospital, Medical Sciences/University of Tehran

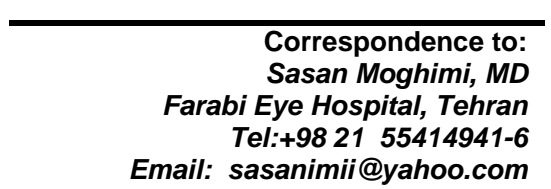

4. Professor of Ophthalmology, Eye Research Center, Farabi Eye Hospital, Medical Sciences/University of Tehran

Received: January 18, 2007

Accepted: April 12, 2007 


\section{Introduction}

Homocysteine, is an intermediary metabolite of the essential dietary amino-acid methionine, metabolized by remethylation to methionine or by trans-sulfuration to cysteine. In the remethylation pathway, homocysteine is methylated to methionine by the enzyme methionine synthase. The methyl group is acquired from 5-methyl tetrahydrofolate and Cobalamin (vitamin B12) is essential for this reaction. ${ }^{1,2}$

Mean plasma homocysteine level shows a great inter-country variation ranging from $6 \mu \mathrm{mol} / \mathrm{l}$, in Japan to $13 \mu \mathrm{mol} / \mathrm{l}$, in South Africa. ${ }^{3}$ Hyperhomocysteinemia refers to mild to moderate elevation of the amino acid homocysteine in blood or plasma. It may be caused by genetic determinants, ${ }^{4}$ lifestyle determinants $^{5}$ (diet, coffee consumption), medications $^{6}$ (methotrexate), and clinical conditions $^{7}$ such as inadequate plasma concentrations of folate or B vitamins which are account for nearly two thirds of all cases of hyperhomocysteinemia in adults. ${ }^{8}$

It is now well documented that elevation of total plasma homocysteine level in a fasting state is associated with an increased risk of cardiovascular diseases such as myocardial infarction, ${ }^{9,10}$ stroke, ${ }^{11,12}$ and carotid intimalmedial wall thickening. ${ }^{13}$ Extensive research in the recent decade has shown that hyperhomocysteinemia is an independent risk factor for vascular disease similar to smoking or hyperlipidemia. ${ }^{14,15}$

Recently hyperhomocysteinemia has also been implicated as an important modifiable potentially risk factor for retinal vein occlusions, especially central retinal vein occlusion (CRVO), which is among the most important causes of vision loss. ${ }^{14-22}$ However data from some recent studies do not confirm this association ${ }^{23-28}$ and it has not been reported in Iranian population yet. In a typical daily Iranian diet, the consumption of fresh fruits, vegetables, fresh herbs, tea, bread and rice is comparatively higher than a typical diet in Western Europe and the US. In a recent study, lower homocysteine levels observed in our population as compared to other populations. $^{29}$ The mean plasma homocysteine level in CRVO may also be subsequently lower in our population.

Thus we conducted a study to evaluate total plasma homocysteine level during acute phase of CRVO compared with a matched healthy group in Iranian population, to determine whether hyperhomocysteinemia is a risk factor for CRVO.

\section{Methods}

We conducted a case-control study on 105 individuals chosen among patients referred to Farabi Eye Hospital over a period of two years (March 2003 to February 2005). The study was reviewed and approved by the ethics committee of the Medical Faculty of Tehran University of medical sciences. Participants had no history of major systemic illness, evidence of vasculitits, renal, hepatic or thyroid disease, cardiomyopathy, pregnancy, and chronic alcohol abuse.

The study group contains 54 patients presenting with CRVO in recent one month, acute phase of the disease. CRVO was considered to be present if there was intra retinal hemorrhage in all four retinal quadrants surrounding the optic disk and scattered up to the periphery with venous dilation and tortousity. Control subjects were adult patients who evaluated in the same clinic for a nonretinal disease diagnosis like cataract and nasolacrimal duct obstruction. Controls were matched the study group patients in potential confounding factors such as age, sex, systemic hypertension, diabetes mellitus, and smoking states. A written informed consent was taken before blood sampling for measurmrnt of total plasma homocysteine level in this group.

The patients received complete ophthalmic and medical examinations, as well as laboratory evaluation including clotting tests. Fasting total plasma homocysteine level was measured in all patients by high-performance liquid chromatography. Hyperhomocysteinemia was defined as a total plasma homocysteine level above $15 \mu \mathrm{mol} / \mathrm{l}$.

Descriptive statistics was used to report demographic characteristics; by the means of SPSS statistic package version 13.5. Independent sample t-test and chi-square test were also used to investigate the significance of quantitative and qualitative difference between homocystein levels in two groups. Logistic regression was used to analyze associations between total plasma homocysteine and CRVO. The adjusted odds 
ratio was calculated as an estimate of the relative risk of CRVO for individuals with hyperhomocysteinemia. Multivariable-adjusted analyses controlled for statistically significant confounding variables of age, sex, hypertension, smoking history and serum creatinine, cholesterol, and triglyceride.

\section{Results}

Fifty four patients (22 females, 32 males) with CRVO were enrolled in the study. Control group included 51 individuals (22 females, 29 males). Analyses were performed on 105 participants with complete information on CRVO status and total plasma homocysteine level. Age and sex were not significantly different in two groups. Potential confounding risk factors such as diabetes mellitus, hypertension, smoking, and serum creatinine, cholesterol, and triglyceride levels were also compared between two groups. Patient's characteristics are shown in the table 1.

The mean total plasma homocysteine level was $14.76 \pm 7.67 \mu \mathrm{mol} / /$ in cases, and $11.42 \pm 3.74 \mu \mathrm{mol} / \mathrm{l}$ in control subjects. It showed a significant difference $(p=0.005)$ in mean plasma homocysteine level between the cases and control group. 17 of 54 patients $(31.5 \%)$ who were presented with CRVO, had total plasma homocysteine above $15 \mu \mathrm{mol} / \mathrm{l}$, but only 7 of $51(13.7 \%)$ control subjects. This difference was statistically significant $(p=0.03)$

Total homocysteine level above $15 \mu \mathrm{mol} / \mathrm{I}$ was nearly 2.5 times more common in patients with CRVO compared with healthy controls. In univariate analyses, odds ratio (OR) of CRVO for individuals with hyperhomocysteinemia was $2.88 ; \quad(95 \%$ $\mathrm{Cl}=1.08-7.71$ and $\mathrm{p}=0.03$ ).

Adjusted odds ratios of different variables are shown in table 2. The overall multivariable-adjusted odds of CRVO in participants with plasma homocysteine level above $15 \mu \mathrm{mol} / \mathrm{l}$ was 4.71 ; $(95 \% \mathrm{Cl}=1.46$ 15.19 and $p=0.009)$. Each $1 \mu \mathrm{mol} / \mathrm{l}$ increase in homocysteine level was associated with significant increased odds for CRVO 0.87; (95\% Cl=0.79-0.97, $\quad \mathrm{p}=0.01$ ). Hyperhomocysteinemia was not statistically different in each age group ( $<60$ years: $27 \%$, $61-70$ years: $33.3 \%, 71-80$ years: $31.6 \%, .>81$ years: $33.3 \%$, Chi-square test, $p=0.98$ ).

Table 1. Descriptive data of CRVO and control subjects

\begin{tabular}{|c|c|c|c|}
\hline & CRVO Group & Controls & $p$ value \\
\hline Number & 54 & 51 & \\
\hline Age (year) & $59.8 \pm 12.7(22-87)$ & $63.0 \pm 8.7(41-78)$ & 0.13 \\
\hline Male sex (\%) & $32(59.3)$ & $29(56.9)$ & 0.80 \\
\hline Hypertension (\%) & $16(29.4)$ & $16(31.4)$ & 0.84 \\
\hline Diabetes (\%) & 7.0 & 5.1 & 0.75 \\
\hline Hematocrit (\%) & $43.1 \pm 4.2(30-52)$ & $43.8 \pm 3.5(34-69)$ & 0.36 \\
\hline Creatinine (mg/dl) & $0.98 \pm 0.17(0.7-1.5)$ & $1.00 \pm 0.23(0.7-1.8)$ & 0.70 \\
\hline Triglyceride (mg/dl) & $189.6 \pm 73.4(57-462)$ & $194.2 \pm 69.9(111-400)$ & 0.76 \\
\hline Cholesterol (mg/dl) & $201.7 \pm 42.2(115-309)$ & $185.9 \pm 41.6(123-173)$ & 0.07 \\
\hline Smoking (\%) & $9(16.7)$ & $5(9.8)$ & 0.30 \\
\hline $\begin{array}{l}\text { Homocysteine level } \\
(\mu \mathrm{mol} / \mathrm{L})\end{array}$ & $14.76 \pm 7.6(5-38)$ & $11.40 \pm 3.70(6.2-23.9)$ & 0.005 \\
\hline $\begin{array}{l}\text { Homocysteine level >15 } \\
\mu \mathrm{mol} / \mathrm{L}(\%)\end{array}$ & $17(31.5)$ & $7(13.5)$ & 0.03 \\
\hline
\end{tabular}


Table 2. Adjusted Odds ratio of different risk factors in patient with CRVO

\begin{tabular}{lccc}
\hline & Adjusted Odds ratio & Confidence interval & p value \\
\hline Hypertension & 1.72 & $0.54-5.41$ & 0.35 \\
Diabetes & 1.55 & $0.16-14.67$ & 0.70 \\
Hematocrit & 1.11 & $0.97-1.27$ & 0.36 \\
Creatinine & 1.27 & $0.94-17.24$ & 0.85 \\
$\begin{array}{l}\text { Triglyceride } \\
\text { Cholesterol }\end{array}$ & 1.00 & $0.99-1.01$ & 0.51 \\
$\begin{array}{l}\text { Smoking } \\
\text { Homocysteine } \\
\text { level }>15 \mu \mathrm{mol} / \mathrm{L}\end{array}$ & 0.99 & $0.98-1.30$ & 0.38 \\
\hline
\end{tabular}

\section{Discussion}

The result of this study demonstrated a clinically important and statistically significant association between the presence of hyperhomocysteinemia and CRVO. Compared with the control group, mean plasma total homocysteine levels were significantly higher in patients with retinal vascular occlusive disease (11.42 $\mathrm{mmol} / \mathrm{l}$ versus $14.76 \mu \mathrm{mol} / \mathrm{l})$. This is the first study to document that elevated plasma homocysteine level is an independent risk factor for CRVO in Iranian population.

Numerous studies have documented that hyperhomocysteinemia is a risk factor for cardiovascular disease. ${ }^{9-13}$ Elevated total homocysteine is both an independent risk factor for atherosclerotic vascular disease ${ }^{14,15}$ and interacts with other risk factors such as smoking and hypertension to increase cardiovascular disease risk. $^{30}$ The exact mechanism by which high plasma concentrations of homocysteine induce arterial and venous thrombosis is still not clear. There is evidence that homocysteine causes endothelial injury and dysfunction by increasing the production of free radicals generated from the oxidation of homocysteine and subsequent peroxidation. Homocysteine also stimulates the proliferation of vascular smooth muscle cells and inhibits the growth of vascular endothelial cells. This combination could lead to atherosclerosis. Other possible mechanisms of thrombosis in hyperhomocysteinemia include increased platelet adhesiveness, activation of the coagulation cascade, and impaired nitric oxide production from endothelial cells. ${ }^{1,2}$ Because the central retinal vein shares a common, fibrous adventitia with the central retinal artery, it is likely that both arterial and venous diseases could contribute to the development of a CRVO. Any sclerotic thickening of the central retinal artery could easily compress the adjacent central retinal vein and begin the sequence of events that leads to thrombus formation. ${ }^{31}$

The results of the present study are in line with previous recent studies that have recognized hyperhomocysteinemia as a risk factor for retinal vascular occlusive disease especially CRVO. ${ }^{14-22}$ However, most of these studies were retrospective, ${ }^{14,21,22}$ subjects were not fasting at the time of homocysteine measurement, ${ }^{14}$ cases and controls were not fully matched, ${ }^{14,22}$ and homocysteine was not measured at the time of the acute retinal vascular occlusion. ${ }^{14}$ This possible bias would result in lower values for the patients with CRVO. Further studies, including two very recent; found no correlation between CRVO and homocysteine. ${ }^{23-28}$ One recent study suggests that the homocysteine plasma level is not a primary and independent risk factor for CRVO, but is more likely a marker of 
atherosclerosis and the consequence of other well-established risk factors. ${ }^{32}$

Our study adds further support for a cardiovascular risk profile for persons with CRVO in Iranian population. These results also support the necessity of screening for possible hyperhomocysteinemia. in all patients with CRVO

Differing mean plasma homocysteine values for healthy people from various countries have been reported that range from $6 \mu \mathrm{mol} / \mathrm{L}$ in Japan to $13 \mu \mathrm{mol} / \mathrm{L}$ in South Africa. ${ }^{3}$ In 1997, Alfthan et al ${ }^{3}$ reported the mean plasma homocysteine concentrations in 13 different countries which ranged from 7.1 $\mu \mathrm{mol} / \mathrm{L}$ in Germany to $10.7 \mu \mathrm{mol} / \mathrm{L}$ in Finland. The reason for inter-country variations of plasma homocysteine is not clear but Alfthan et $\mathrm{al}^{3}$, argued that these differences are real and not due to non-compatible sampling and assay procedures. A lower homocysteine level reported in our country ${ }^{29}$ is comparable with values obtained in France, Spain and Japan. ${ }^{3}$ Geographical variation in fruit and vegetable intakes and particularly in dietary folate, might explain these inter-country differences. It has been suggested that a high dietary intake of folate can make a substantial contribution to the reduction in total plasma homocysteine concentrations in the general population. ${ }^{29}$ In a typical daily Iranian diet the consumption of fresh fruits, vegetables, fresh herbs, tea, bread and rice is comparatively higher than a typical diet in Western Europe and the US. This might explain the lower homocysteine levels observed in our population as compared to other populations. ${ }^{29}$

According to the lower level of total plasma homocysteine in Iranian population, we expected to obtain lower levels in our study group compared with other countries. But the mean total plasma homocysteine level, neither in cases nor in controls, did not show a significant different compared with other studies. We also failed to find any difference in existence of hyperhomocysteinemia among different ages, where other studies ${ }^{15}$ demonstrated more strongly association in patients younger than 70 years old.
The potential importance of treating underlying cardiovascular risk factors is borne out by several reports. Patients with retinal vascular occlusive disease have a significant excess of mortality from coronary and cerebrovascular diseases. ${ }^{3,33,34}$ Unlike other risk factors, hyperhomocysteinemia is readily reversible in most individuals with supplying inexpensive vitamin preparations containing folic acid and other vitamins. Vitamin supplementation reduces homocysteine level regardless of the underlying cause ${ }^{35,36}$ Folate supplementation is economical and does not give rise to any major side-effects, raising the possibility that some forms of vascular disease may be easily, safely, and inexpensively treated. It is assumed that by reducing the toxic effect of homocysteine on the blood vessels, the probability of further retinal and systemic vascular occlusion, and therefore the resulting morbidity and mortality will be reduced. It has been shown that homocysteine-lowering treatment significantly reduced the risk of vascular events and other sequels of classical hyperhomocysteinemia. ${ }^{36,37}$ However, as it is still controversial, further research to assess this association may be useful. Large-scale randomized trials will be required to confirm that reducing plasma homocysteine levels results in decreased retinal and systemic atherothrombotic vascular disease.

\section{Conclusion}

Elevated total plasma homocysteine level is an independent risk factor for CRVO in Iranian population. In addition to an evaluation of all conventional cardiovascular risk factors, measurement of total homocysteine for evidence of hyperhomocysteinemia may be important in the initial investigation and management of patients with CRVO. It is suggested to conduct a study to evaluate effect of lowering homocysteine levels by vitamin supplementation on prevention of recurrence of disease in the same or fellow eye, reduction of other cardiovascular events, and improvement of the prognosis. 


\section{References}

1. Townend J, O' Sullivan J, Wilde JT. Hyperhomocysteinemia and vascular disease. Blood Rev 1998; 12:23-34.

2. Guba SC, Fink LM, Fonseca V. Hyperhomocysteinemia: an emerging and important risk factor for thromboembolic and cardiovascular disease. Am J Clin Pathol 1996; 105:709-22.

3. Alfthan G, Aro A, Gey KF: Plasma homocysteine and cardiovascular disease mortality. Lancet 1997; 349(9049):397.

4. Frosst $P$, Blom $H J$, Milos $R$, et al. A candidate genetic risk factor for vascular disease: a common mutation in methylenetetrahydrofolate reductase. Nat Genet 1995; 10:111-3.

5. Nygård $\mathrm{O}$, Refsum $\mathrm{H}$, Ueland $\mathrm{PM}$, Vollset SE. Major lifestyle determinants of plasma homocysteine distribution: the Hordaland Homocysteine Study. Am J Clin Nutr 1998; 67:26370.

6. Morgan SL, Baggott JE, Lee JY, Alarcon GS. Folic acid supplementation prevents deficient blood folate levels and hyperhomocysteinemia during long-term, low dose methotrexate therapy for rheumatoid arthritis: implications for cardiovascular disease prevention. J Rheumatol 1998; 25:441-6.

7. Refsum H, Ueland PM, Nygård $\mathrm{O}$, et al. Homocysteine and cardiovascular disease. Ann Rev Med 1998; 49:31- 62.

8. Selhub J, Jacques PF, Wilson PW, et al. Vitamin status and intake as primary determinants of homocysteinemia in an elderly population. JAMA 1993; 270:2693-8.

9. Salahuddin MI, Ahmed SI. Homocysteine level in patients with established transmural myocardial infarction. J Coll Physicians Surg Pak 2005; 15:520-3.

10. Ogawa M, Abe S, Saigo M, et al. Homocysteine and hemostatic disorder as a risk factor for myocardial infarction at a young age. Thromb Res 2003; 109:253-8.

11. Wang $\mathrm{H}$, Fan $\mathrm{D}$, Zhang $\mathrm{H}$, et al. Serum level of homocysteine is correlated to carotid artery atherosclerosis in Chinese with ischemic stroke. Neurol Res 2006; 28:25-30.

12. Virtanen JK, Voutilainen S, Happonen P, et al. Serum homocysteine, folate and risk of stroke: Kuopio Ischaemic Heart Disease Risk Factor (KIHD) Study. Eur J Cardiovasc Prev Rehabil 2005; 12:369-75.

13. Malinow MR, Nieto FJ, Szklo M, et al. Carotid artery intimal-medial wall thickening and plasma homocyst(e)ine in asymptomatic adults: the Atherosclerosis Risk in Communities Study. Circulation 1993; 87:1107-13.

14. Vine AK. Hyperhomocysteinemia: a risk factor for central retinal vein occlusion. Am J Ophthalmol 2000; 129:640-4.

15. Chua B, Kifley A, Wong TY, Mitchell P. Homocysteine and retinal vein occlusion: a populationbased study. Am J Ophthalmol 2005; 139:181-2.

16. Abu El-Asrar AM, Abdel Gader AG, Al-Amro SA, Al-Attas OS. Hyperhomocysteinemia and retinal vascular occlusive disease. Eur J Ophthalmol 2002; 12:495-500.

17. Terrazzi P, Di Micco P, Quaglia I, et al. Homocysteine, MTHFR C677T gene polymorphism, folic acid and vitamin B12 in patients with retinal vein occlusion. Thromb J 2005; 3:13.

18. Yildirim C, Yaylali V, Tatlipinar S, et al. Hyperhomocysteinemia: a risk factor for retinal vein occlusion. Ophthalmologica 2004; 218:102-6.

19. Weger M, Stanger O, Deutschmann H, et al. Hyperhomocyst(e)inemia and MTHFR C677T genotypes in patients with central retinal vein occlusion. Graefes Arch Clin Exp Ophthalmol 2002; 240:286-90.

20. Cantu C, Alonso E, Jara A, et al. Hyperhomocysteinemia, low folate and vitamin B12 concentrations, and methylene tetrahydrofolate reductase mutation in cerebral venous thrombosis. Stroke 2004; 35:1790-4.

21. Cahill M, Karabatzaki M, Donoghue $C$, et al. Thermolabile MTHFR genotype and retinal vascular occlusive disease. Br J Ophthalmol 2001; 85:88-90.

22. Martin SC, Rauz S, Marr JE, Martin N, Jones AF, Dodson PM. Plasma total homocysteine and retinal vascular disease. Eye 2000; 14:590-3. 
23. Boyd S, Owens D, Gin T, et al. Plasma homocysteine, methylene tetrahydrofolate redoctase C677T and factor II G20210A polymorphisms, factor VIII, and VWF in central retinal vein occlusion. Br J Ophthalmol 2001; 85:1313-5.

24. Pianka $\mathrm{P}$, Almog $\mathrm{Y}$, Man $\mathrm{O}$, et al. Hyperhomocystinemia in patients with nonarteritic anterior ischemic optic neuropathy, central retinal artery occlusion and central retinal vein occlusion. Ophthalmology 2000; 107:1588-92.

25. Larsson J, Hultberg B, Hillarp. Hyperhomocysteinemia and the MTHF C677T mutation in central retinal vein occlusion. Acta Ophthalmol Scand 2000; 78:340-3.

26. Pinna A, Carru C, Zinellu A, Dore S, Deiana L, Carta F. Plasma homocysteine and cysteine levels in retinal vein occlusion. Invest Ophthalmol Vis Sci 2006; 47:4067-71.

27. McGimpsey SJ, Woodside JV, Bamford L, Gilchrist SE, Graydon R, McKeeman GC, et al. Retinal vein occlusion, homocysteine, and methylene tetrahydrofolate reductase genotype. Invest Ophthalmol Vis Sci 2005; 46:4712-6.

28. Di Crecchio L, Parodi MB, Sanguinetti G, lacono P, Ravalico G. Hyperhomocysteinemia and the methylenetetrahydrofolate reductase $677 \mathrm{C}-\mathrm{T}$ mutation in patients under 50 years of age affected by central retinal vein occlusion. Ophthalmology. 2004;111:940-5.

29. Golbahar J, Bararpour $\mathrm{H}$. Normal range of total plasma homocysteine concentrations in southern Iran. IJMS 2003; 28:139-42.

30. Graham IM, Daly LE, Refsum HM, et al. Plasma homocysteine as a risk factor for vascular disease. The European Concerted Action Project. JAMA 1997; 277:1775-81.

31. Green WR, Chan CC, Hutchins GM, Terry JM. Central retinal vein occlusion: a prospective histopathologic study of 29 eyes in 28 cases. Trans Am Ophthalmol Soc 1981; 79:371- 422.

32. Parodi MB, Di Crecchio L. Hyperhomocysteinemia in central retinal vein occlusion in young adults. Semin Ophthalmol 2003; 18:154-9.

33. Cugati S, Wang JJ, Knudtson MD, et al. Retinal vein occlusion and vascular mortality pooled data analysis of 2 population-based cohorts. Ophthalmology 2007; 114:520-4.

34. Tsaloumas MD, Kirwan J, Vinall $\mathrm{H}$, et al. Nine year follow-up study of morbidity and mortality in retinal vein occlusion. Eye 2000; 14:821-7.

35. Van der Griend R, Haas FJ, Biesma DH, et al. Combination of low-dose folic acid and pyridoxine for treatment of hyperhomocysteinemia in patients with premature arterial disease and their relatives. Atherosclerosis 1999; 143:177-83.

36. Kluijtmans LA, Boers GH, Kraus JP, et al. The molecular basis of cystathionine beta-synthase deficiency in Dutch patients with homocystinuria: effect of CBS genotype on biochemical and clinical phenotype and on response to treatment. Am J Hum Genet 1999; 65:59-67.

37. Miller JW. Does lowering plasma homocysteine reduce vascular disease risk? Nutr Rev 2001; 59:242-4. 